\title{
Evaluation of a health sciences internship for Latino and Native American library students
}

\author{
Alla Keselman, PhD, MA; Sanjana Quasem, BS; Janice E. Kelly, MLS; Gale A. Dutcher, MS, MLS, AHIP \\ See end of article for authors' affiliations. \\ DOI: http://dx.doi.org/10.3163/1536-5050.104.4.016
}

Purpose: This paper presents a qualitative evaluation of a graduate-level internship for Latino and Native American library science students or students who are interested in serving those populations.

Methods: The authors analyzed semi-structured interviews with thirteen internship program graduates or participants.

Results: The analysis suggests that the program increased participants' interest in health sciences librarianship and led to improved career opportunities, both in health sciences libraries and other libraries with health information programming. It also highlights specific factors that are likely to contribute to the strength of career pipeline programs aiming to bring Latino and Native American students and students who are interested in serving those communities into health librarianship.

Conclusions: Exposing graduate-level interns to a broad range of health sciences librarianship tasks, including outreach to Latino and Native American communities and formal mentorship, is likely to maximize interns' interests in both health sciences librarianship and service to these communities.

Keywords: Internships and Residency; Librarians; Libraries, Medical; Library Science/Education; Program Evaluation

\section{PROGRAM BACKGROUND}

Reducing health disparities by improving health information access for underserved minorities and health professionals who serve them has long been one of the key objectives of health librarianship [1]. A related objective is to attract members of underrepresented minority groups into the library field. Latinos and Native Americans are currently the most underrepresented minority groups in library and information science [2].

This case study presents an evaluation of and lessons learned from a graduate-level professional development and tuition assistance internship at the University of Arizona Health Sciences Library (UAHSL) for Latino and Native American students or for students who are interested in serving those populations. The internship, sponsored by the US National Library of Medicine (NLM), is available to library science students enrolled in the Knowledge River (KR) program, an educational fellowship for students committed to addressing the information needs of Latino and Native American populations $<$ https://si.arizona.edu/knowledge-river-0 $>$.

UAHSL serves the University of Arizona Colleges of Medicine, Nursing, Pharmacy, and Public Health, and works in partnership with Banner-University Medical Center (a teaching hospital). In addition, its resources and services, including extensive outreach programs, are available to community members statewide. Between 2003 and 2015, NLM support enabled UAHSL to provide yearlong internship placements to twelve cohorts of twenty-five KR students, with one to three students participating each year. The objectives of the internships are to attract KR students to health sciences librarianship, improve their understanding of the field by giving them practical experience, and expose them to career opportunities. UAHSL also feels that KR students would enrich the ongoing UAHSL outreach projects with their cultural and linguistic expertise. 


\section{EVALUATION OBJECTIVES}

The authors sought to determine the impact of the internship on participants':

1. awareness of the field of health sciences librarianship and understanding of career opportunities,

2. interest in careers in medical and health information librarianships, and

3. actual career trajectories.

In addition, we were interested in exploring how participants' interest in serving Latino and Native American communities interacted with their internship experiences.

\section{METHODOLOGY}

The protocol was exempt from internal review board review by the National Institutes of Health (NIH) Office of Human Subjects Research.

\section{Recruitment}

A list of twenty-five program participants, including their email addresses at the time of graduation, was obtained from UAHSL. One of the authors (Quasem) emailed all participants at the provided email addresses, as well as addresses found via Internet searches. Thirteen program participants responded and agreed to participate. The authors could not determine how many of the remaining twelve were not reached because inquiries were sent to outdated addresses and how many chose not to respond.

\section{Participants}

Of the thirteen, one participant each were from the fourth and fifth cohorts; two participants each were from the seventh, eleventh, and twelfth cohorts; and three participants were from the eighth cohort. Nine participants self-identified as Latino and two as Native Americans. Five were male, and eight were female. One author (Quasem) also conducted telephone interviews with the UAHSL director and deputy director, who were instrumental in establishing and maintaining the program.

\section{Protocol}

The semi-structured interview guide for the interns focused on their pre-internship backgrounds (educational and professional experience, career plans, awareness of health sciences librarianship, interest in working with Native Americans and Latinos, awareness of health concerns in Latino and Native American communities); internship experience (tasks; interests, knowledge, and skills acquired; networking and mentorship; perceived strengths and limitations of the internships); and post-internship experience (career trajectory). The interview guide for the program administrators focused on the program's background and history.

\section{Analysis}

All interviews were audio-recorded and transcribed, and coded using NVivo 10 (QSR; Sydney, Australia), drawing on a previously described approach [3]. Two authors coded a subset of protocols, verifying satisfactory level of inter-coder agreement. Following that, one author coded the remaining protocols.

\section{RESULTS}

\section{Professional trajectory into health careers}

Prior to the internship, participants may have had an interest in health, but little knowledge of health sciences librarianship. Of the thirteen, none entered library school with the goal of becoming a health librarian. Seven did not name a specific library setting when discussing their pre-internship professional plans; two wished to become public librarians; the others mentioned various specialized library settings (e.g., an academic library, a school library). At the time of the study, one was completing the second year of the master's of library science (MLS) program, while twelve had graduated. Of these twelve, three worked in health sciences libraries; two were community outreach specialists; and one was a biomedical librarian. Another four were librarians in community colleges with allied health programs, and all of the four were involved in health information provision or programming to some extent. Of the five graduates who were not employed in health or academic library settings, one was a public librarian in a small rural library, two were doctoral students in library science, one worked as a librarian at a major national park library, and one was an archivist at an archeological conservation center.

While not all participants became health librarians, most developed a positive view of health librarianship. Three participants who did not work 
as health sciences librarians at the time of the interview stated that they had applied for or contemplated applying for such jobs during their career. Only two expressed no interest in health sciences librarianship as a field. Of these, one started the program with a very clear goal of becoming a tribal archivist and was able to achieve that goal. The other, however, explained her lack of interest in the field by saying, "I didn't feel like I learned that much that made me want to be a medical librarian."

The two program administrators who were interviewed reported that three former interns later worked as UAHSL librarians. Another three became NLM Associate Fellows. While the administrators explained that they did not follow the careers of most participants, they told stories of two interns who, upon becoming public librarians, went on to establish strong consumer health programs in their libraries.

\section{Health information needs of Latino and Native American communities and health librarianship}

As KR fellows, all interns came to library school with a dedication to serving Latino and Native American communities in some capacity. Most also had firsthand personal experiences with health disparities. For example, participant 13 said, "Well, being Latina, I knew that there were a lot of disparities. I knew a lot of minorities could not afford healthcare and relied on folk medicine. Some had problems with the language barrier." Participants also knew about disparities in health knowledge and outcomes from their MLS coursework and, in some cases, pre-MLS work experience. However, their understanding of the specific role librarianship could play in reducing health disparities was limited.

Two of the thirteen participants felt that their UAHSL internship provided them with a comprehensive exposure to health information needs of Latinos and Native Americans and the role of librarianship in addressing these concerns. Both of these participants assisted in developing consumer health information resources as part of their internships; one also participated in conducting outreach events for Latinos and Native Americans. Other participants felt that addressing health information needs of Latinos and Native Americans was not the focus of their internships. However, several participants mentioned conversations with librarians or other interns who were involved in outreach projects, as well as exposure to a diverse range of library patrons.

\section{Perceived benefits of the internship}

Skills and knowledge. In the course of the internship, participants gained professional skills. Most gained experience with reference desk work, including an opportunity to interact with patrons, conduct reference interviews, and search databases for patrons or resource development. Many also gained experience with cataloging, classification, interlibrary loan, and digital services. Several participated in preparing presentations. Direct experience with exhibiting and conducting outreach was relatively rare, described by only three participants. In addition to specific skills, participants also gained an understanding of library operations and different types of library services.

\section{Confidence, resume-building, and professional} connections. Many participants felt that the internship furthered their careers. Importantly, the internship provided them with specific tangible skills and resume-strengthening experience. In addition, a number of participants commented how the internship gave them professional confidence and equipped them with a network and connections for job searches. In the words of participant 1, "I had a refined skillset, gained confidence, and, upon graduating, felt like I had enough experience to sell myself to someone where I had to show I was successful." Participant 4 discussed how the internship enabled her to obtain a very competitive NLM Associate Fellowship. Two more participants commented that their postUAHSL employment came as a result of UAHSL internship networking.

Relationship with library staff. While the internship did not formally assign mentors to interns, many participants commented that the relationship with UAHSL librarians and library staff was the most positive aspect of the internship. For example, participant 1 said, "[Names of two librarians] were very. . .generous of their time. I asked them about their career paths and working conditions." Participant 10 described her relationship with the same librarians as follows: "I was very fortunate. ..to establish the mentor-mentee relationship with them. Not only my internship, but they were concerned with my classes. They were just very helpful."

Almost all participants described librarians and library staff as approachable and encouraging. In 
addition to providing specific tasks and projects, the librarians discussed different career paths and professional opportunities with the interns. Some also helped with mock interviews and provided professional references. However, several participants commented that while the librarians were supportive, they were often too busy, because the libraries were short-staffed. Participant 6 explained that "quality was there, [but]...the availability was a difficult thing." One participant felt that library staff "did not offer support to Knowledge River students," because they were either too busy or felt that the internship was too short for a useful student engagement. One student who, because of her schedule, chose to complete her internship during the evening hours, commented that there were no librarians, only paraprofessionals, on staff when she was at the library.

Attention to students' interests. In discussing the program's strengths, five participants commented on the freedom that was awarded to them in the choice of their tasks and on the UAHSL librarians' attempts to accommodate their interests. For example, participant 1 commented on the "openness in crafting daily experience according to what skillset I was interested in developing." Participant 4 said, "I had a lot of supervision and support, but my supervisors allowed me to complete this project in a very independent way."

Attending the Medical Library Association annual meeting. Eleven of the thirteen participants were able to attend the Medical Library Association (MLA) annual meeting as part of their internship (two could not go for personal reasons). Of these, all but one (who did not recall much from the meeting) spoke of the meeting as extremely useful and influential in their professional development. The benefits came from attending workshops and sessions, as well as from networking and gaining full exposure to the universe of health sciences librarianship. For example, to participant 8 , the MLA meeting "opened up a plethora of information," including about databases and new technologies. Participants 1 and 2 talked about meeting health sciences librarians from across the country. Participant 4 called the experience "very meaningful," adding, "I had a chance to get an idea of what people were looking at and doing beyond what I was doing at the library." Several participants described their MLA attendance as one of the best experiences of their internship.
Practical considerations. Participants mentioned other strengths of the internship, including the stipend (e.g., one participant specifically commented on how she would not have been able to attend library school otherwise) and flexible hours that allowed them to combine the internship with their classes.

\section{Participants' recommendations for strengthening the program}

A common request was to make the program more structured with more tasks preplanned, rather than ad hoc, thus creating an evenly distributed workload and eliminating stretches of inactivity. Several participants encouraged regular supervision or check-ins on the progress of their work, as well as periodic formal evaluations with bidirectional feedback. Several also asked for more formal mentorship.

While most participants had a very positive view of the program, three felt that the tasks in which they were engaged were administrative or clerical in nature (e.g., handling keys for study rooms), which did not utilize their professional development potential. This was partly related to the somewhat ad hoc nature of the internship and partly to these participants' work schedules: all three worked evening shifts, when the library was staffed by paraprofessionals. Expressing her wish for more meaningful tasks, participant 4 explained, "We had a lot of unhappy KR students go through the program and not gain much. I think there is so much potential, and it's the only health sciences library in Tucson that is willing to work with Knowledge River, but something needs to be changed to give students meaningful work in an area they would excel." Similarly frustrated, participant 13 (who worked night shifts because of her school schedule) said, "If I was put to actively work with librarians, rather than set out as a clerk, I would have gotten a lot more out of it."

In making suggestions about tasks for future interns, almost all participants asked for a rotation among different assignments. While several commended the library for making an effort to match tasks to interns' interests, they also pointed out that students did not always know what additional things could spark their interest, given exposure. Students particularly asked for additional experience with outreach. 
Finally, some students suggested that UAHSL could help interns with exposure to different models of health sciences librarianship and different types of health sciences libraries (e.g., hospital libraries).

\section{LESSONS LEARNED}

This evaluation suggests that providing financial and professional development support in a health sciences library to Latino and Native American library students increases their interest in health sciences librarianship and leads to improved career opportunities, both in health sciences libraries and in other libraries that conduct health information programming. The experience is strengthened by attention to students' interest, exposure to a range of professional tasks, and MLA annual meeting attendance. The following steps are likely to further improve interns' development:

1. Providing more targeted experience in serving information needs of Latino and Native American communities and their health service providers may strengthen the draw of health sciences librarianship as the means for serving those communities.

2. Structuring an internship as a rotation among different predefined tasks that involve outreach, as well as more technical aspects of library science, is likely to provide a comprehensive perspective of the field and help students discover new professional interests. This will also eliminate ad hoc tasks that may meet the internship site's needs, but do not support professional development.

3. Insisting that students work during regular business hours will take away some scheduling flexibility, but will also ensure optimal opportunities for interacting with professional librarians.

4. Assigning formal mentors and providing incentives for mentoring will strengthen interactions and learning exchanges between students and experienced librarians.

5. Exposing interns to different types of health sciences library settings can further strengthen interest in the field.

Additionally, recruiting students who are either already interested in health sciences librarianship or have broad and undefined interests is more likely to encourage the students into careers with a health information focus and is preferable to recruiting students with strongly specialized but not healthrelated interests.

\section{ACKNOWLEDGMENT}

The authors thank Jeanette Ryan and Gerald J. Perry, AHIP, for their comments on an earlier version of this manuscript. We are also grateful to the participants for their time and thoughts. This work was supported by intramural research program of the NLM, NIH.

\section{REFERENCES}

1. National Library of Medicine Board of Regents. Charting a course for the 21st century: NLM's long range plan 2006-2016 [Internet]. (NIH publication no.07-4890). National Institutes of Health; 2006 [cited 6 Jan 2016]. 66 p. $<$ http://www.nlm.nih.gov/pubs/plan/>.

2. American Library Association. Diversity counts [Internet]. The Association [cited 6 Jan 2016]. <http:// www.ala.org/offices/diversity/diversitycounts/ divcounts $>$.

3. Keselman A, Ahmed EA, Williamson DC, Kelly JE, Dutcher GA. Harnessing health information to foster disadvantaged teens' community engagement, leadership skills, and career plans: a qualitative evaluation of the Teen Health Leadership Program. J Med Libr Assoc. 2015 Apr;103(2): 82-6. DOI: http://dx.doi.org/10.3163/15365050.103.2.005.

\section{AUTHORS' AFFILIATIONS}

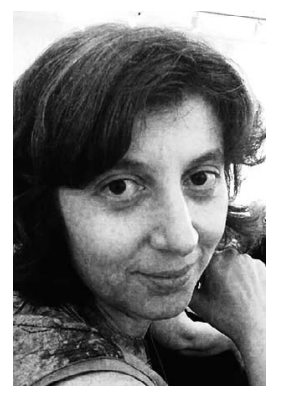

Alla Keselman, PhD, MA (corresponding author), keselmana@ mail.nih.gov, Division of Specialized Information Services, National Library of Medicine, 6707 Democracy Boulevard, Suite 510, Bethesda, MD 20892; Sanjana Quasem, BS, Asian American Health Initiative, Montgomery County Department of Health \& Human Services, 1335 Piccard Drive, Rockville, MD 20850; Janice E. Kelly, MLS, Division of Specialized Information Services, National Library of Medicine, 6707 Democracy Boulevard, Suite 510, Bethesda, MD 20892; Gale A. Dutcher, MS, MLS, AHIP, Division of Specialized Information Services, National Library of Medicine, 6707 Democracy Boulevard, Suite 510, Bethesda, MD 20892

Received March 2016; accepted May 2016 